\title{
Adaptation to a bacterial pathogen in Drosophila melanogaster is not aided by sexual selection
}

\author{
Sakshi Sharda ${ }^{1}$, Tadeusz Kawecki ${ }^{1}$, and Brian Hollis ${ }^{2}$ \\ ${ }^{1}$ University of Lausanne \\ ${ }^{2}$ University of South Carolina
}

August 10, 2021

\begin{abstract}
Theory predicts that sexual selection should aid adaptation to novel environments, but empirical support for this idea is limited. Pathogens are a major driver of host evolution and, unlike abiotic selection pressures, undergo epidemiological and co-evolutionary cycles with the host involving adaptation and counteradaptation. Because of this, populations harbor ample genetic variation underlying immunity and the opportunity for sexual selection based on condition-dependent "good genes" is expected to be large. In this study, we evolved populations of Drosophila melanogaster in a 2-way factorial design manipulating sexual selection and pathogen presence, using a gram-negative insect pathogen Pseudomonas entomophila, for 14 generations. We then examined how the presence of sexual selection and the pathogen, as well as any potential interaction, affected the evolution of pathogen resistance. We found increased resistance to P. entomophila in populations that evolved under pathogen pressure, driven primarily by increased female survival after infection despite selection for resistance acting only on males over the course of experimental evolution. This result suggests that the genetic basis of resistance is in part shared between the sexes. We did not find any evidence of sexual selection aiding adaptation to pathogen, however, a finding contrary to the predictions of "good genes" theory. Our results therefore provide no support for a role for sexual selection in the evolution of immunity in this experimental system.
\end{abstract}

\section{Introduction}

Darwin posited that sexual selection plays an important role in improving non-sexual fitness, writing that, "the strongest and most vigorous males, or those provided with the best weapons, have prevailed under nature, and have led to the improvement of the natural breed or species" (Darwin, 1871). The modern version of this idea proposes that sexually selected traits in males reflect "good genes" (Fisher, 1930; Zahavi, 1975; Iwasa et al. , 1991; Houle \& Kondrashov, 2002), explaining potentially costly female choice by indirect benefits received in the form of increased offspring fitness. Theory suggests expression of sexually-selected traits should evolve to become dependent on overall condition - which would maintain signal fidelity-leading to accelerated rates of adaptation (Lorch et al. , 2003) and more efficient purging of deleterious mutations (Whitlock \& Agrawal, 2009).

In line with predictions of positive effects of sexual selection on population performance, sexual selection has been found to diminish the likelihood of population extinction (Jarzebowska \& Radwan, 2010; Lumleyet al. , 2015). Experimental work in Drosophila has also shown that the presence of sexual selection accelerates the purging of deleterious alleles in experimental populations (Radwan, 2004; Holliset al. , 2009; Whitlock \& Agrawal, 2009; Grieshop et al. , 2016). In several experiments, sexual selection facilitated adaptation to novel environmental challenges, including the evolution of desiccation resistance in D. melanogaster (Gibson Vega et al. , 2020), pesticide resistance in Tribolium castaneum (Jacombet al. , 2016), and adaptation to a novel diet in Callosobruchus maculatus (Fricke \& Arnqvist, 2007). However, an arguably larger body of 
experimental work has found no role for sexual selection in improving non-sexual fitness. Multiple experimental evolution studies failed to find population-level net benefits of sexual selection when examining larval competitive ability, net reproductive rate, or female fecundity (Promislow et al. , 1998; Holland \& Rice, 1999; Long et al. , 2009 respectively). Moreover, a large body of work has also failed to demonstrate a role of sexual selection in adaptation in novel environments (e.g. to higher temperatures (Holland, 2002) or a novel diet (Rundle et al. , 2006)). There is also no evidence that overall mutation load from the genome is reduced under heightened sexual selection (Hollis \& Houle, 2011; Arbuthnott \& Rundle, 2012) (although in environments that are spatially complex, this is not true and the predicted beneficial effects of sexual selection on mutation load are seen (Singh et al. , 2017)). Thus, taken together, the literature is equivocal about role of sexual selection in non-sexual fitness. This leaves an open question about whether the "good genes" mechanism plays a role in adaptation in general or even in specific scenarios, like during adaptation to pathogens or parasites, where this role has been predicted to be most evident but remains largely untested.

One potential explanation for these mixed results is that the non-sexual fitness of populations is normally elevated by competition for mates-that is, sexual selection in the broad sense does have adaptive valuebut these benefits are counterbalanced by the negative effects of sexual conflict and therefore invisible in many experimental designs. Sexual conflict arises because of an evolutionary conflict of interests between the sexes (Parker, 1979; Hosken et al. , 2019) which can manifest in two ways. The first, interlocus sexual conflict, is characterized by selection favoring traits that increase male competitive success even when these traits are accompanied by harm to females. Interlocus sexual conflict can lead to the evolution of female resistance and sexually antagonistic coevolution (Holland \& Rice, 1999a; Chapman et al. , 2003; Rice et al. , 2006), reducing mean population fitness (Bonduriansky \& Chenoweth, 2009; Long et al. , 2009, 2012). In Drosophila, interlocus sexual conflict acts through antagonistic effects on female fecundity and survival (Rice, 1996; Chapman, 2006), especially on the most fecund females (Longet al. , 2009). Intralocus conflict, on the other hand, involves sexually antagonistic pleiotropic effects of polymorphisms at the same locus in males and females (Bonduriansky \& Chenoweth, 2009; Van Doorn, 2009; Innocenti \& Morrow, 2010)) that constrain males and females from reaching sex-specific optima (Chippindale, 2001; Hollis et al. , 2014, 2019). Either form of sexual conflict leads to a burden on populations that might overwhelm any positive effects of sexual selection for mean population fitness (Bonduriansky \& Chenoweth, 2009; Long et al. , 2009, 2012).

Male-male competition and female choice have been proposed to be particularly consequential for evolution of pathogen resistance (Hamilton \& Zuk, 1982; Folstad \& Karter, 1992; Roberts et al. , 2004). Pathogens are a major evolutionary driver of the life histories of organisms (Price, 1980; Schmid-Hempel, 2005) due to their prevalence, diversity, and because they adapt to the host and represent a moving target for the immune system. According to the Hamilton-Zuk hypothesis (1982), sexual ornaments indicate immunity towards prevalent pathogens or parasites (Hamilton \& Zuk, 1982; Martin, 1990). A number of studies in birds have indeed demonstrated phenotypic correlations between male parasite or pathogen load and the quality of sexual ornaments (Hamilton \& Zuk, 1982; Martin, 1990; Balenger \& Zuk, 2014) or female preference towards the males (Blount et al. , 2003; Hund et al. , 2020). Yet, whether this phenotypic correlation should be positive or negative is not unequivocally predicted by mathematical models; either may be predicted depending on details of the model assumptions (Getty, 2002). These phenotypic correlations between sexual ornaments and parasite/pathogen resistance do not necessarily predict whether sexually attractive fathers will sire resistant offspring; rather, this key element of the "good genes" hypothesis is mediated by additive genetic correlations (Hamilton \& Zuk, 1982). One way to test for this genetic correlation would be to track the evolution of resistance under controlled laboratory conditions (Kawecki et al. , 2012) where both the strength of sexual selection and pathogen pressure are manipulated. If there is an additive genetic correlation between sexually successful fathers and pathogen-resistant offspring, resistance should evolve more readily in populations where males also experience sexual selection.

Selection for improved immunity (including better physiological responses to immune challenges) in experimental populations has generally resulted in a robust and rapid response (Armitage \& Siva-Jothy, 2005; Martins et al. , 2013; Joop et al. , 2014; Ferro et al. , 2019). Two studies that explored the effect of sexual selection on immunity by experimentally evolving populations with and without sexual selection have 
found that males and females diverge in their investment in innate immunity (measured as phenyloxidase activity; PO) (Hangartner et al. , 2015; Bagchi et al. , 2021). In both these studies (one on the flour beetle Tribolium castaneum and the other on the seed beetle Callosobruchus maculatus; (Hangartner et al. , 2015; Bagchi et al. , 2021 respectively)), females from polygamous populations had higher levels of PO than females from monogamous populations, with no effect on males from either of the two experimental regimes. The higher levels of PO in females from sexually selected populations did not influence pathogen clearance in either study, although in one of the studies higher PO activity was correlated with lower survival in females upon bacterial infection (Bagchi et al. , 2021). These studies indicate how sexual selection and sexual conflict can drive sex-specific differences in male and female immunity. This pattern is not without exceptions; a study on the yellow dung fly, Scathophaga stercoraria, did not report sex differences in PO levels in populations evolved with or without sexual selection (Hosken 2001). Hosken (2001) also found that monogamous populations had higher PO levels than polygamous populations, although here also this difference did not translate into differences in bacterial clearance after infection (Hosken, 2001). The above studies manipulated the presence or absence of either a pathogen or sexual selection. In the work reported here, we manipulated both pathogen and sexual selection in order to test for effects of the presence of each, as well as any interaction, on the evolution of pathogen resistance.

We carried out a 2-way factorial evolutionary experiment manipulating sexual selection and exposure to a pathogen. We let replicate populations of D. melanogaster evolve for 14 generations either under controlled monogamy or random polygamy (i.e., with or without sexual selection (Hollis \& Houle, 2011)), each generation exposing males to either an intestinal pathogen (a gram-negative bacteriumPseudomonas entomophila ) or a sham treatment. In our experimental design, we only exposed males to the pathogen and allowed the males to interact with females after one day of exposure to the pathogen (we verified that males had cleared the bacteria from their gut at this timepoint, and thus did not infect the females). With this design, we aimed to increase the opportunity for sexual selection to act via differential mating success of males differentially coping with infection. We aimed to address several interconnected questions.

First, and most simply, do D. melanogaster populations exposed to the pathogen as adults evolve resistance, measured as survival after infection, over a short timescale? Resistance to P. entomophilahas been reported to evolve after only four generations of strong selection imposed by breeding from flies that survived a prior infection (Martins et al. , 2013). Second, if only one sex-in our design, males-experiences the pathogen, would evolved resistance to $P$. entomophila be detectable in the other sex? If evolved resistance is evident in both sexes, this would indicate a shared genetic basis. Third, would sexual selection lead to the evolution of differences in pathogen resistance even in the absence of pathogen? This would be predicted if there were an additive genetic correlation between male sexual traits and resistance that were expressed irrespective of pathogen exposure (Joye \& Kawecki, 2019). A result supporting this prediction has been reported in Tribolium (Hangartner et al. , 2015) and Callosobruchus (Bagchi et al. , 2021); however, the conclusion was based on quantifying an aspect of immune response rather than resistance to an actual pathogen. Fourth, does sexual selection accelerate the evolution of resistance in populations exposed to the pathogen, and does it do so to a greater degree than would be expected based on the sum of effects of sexual selection and pathogen exposure acting alone? This positive interaction between the effects of pathogen and sexual selection would be expected if heritable variation in pathogen resistance influenced infected males' sexual success.

The rationale of this study relied on the pathogen affecting the sexual success of males. Therefore, prior to the evolutionary experiment we tested if infection with $P$. entomophila affects competitive paternity share. Mortality in our laboratory population was much lower than is generally reported (Martins et al. , 2013; Faria et al. , 2015; Joye \& Kawecki, 2019), but uninfected males had greater competitive paternity success than infected males. If genetic variation conferring resistance to $P$. entomophila has a similar positive effect on male competitive success after exposure to the pathogen, this scenario should provide an opportunity for female choice to amplify nonsexual selection and accelerate adaptation to pathogen.

\section{Materials and Methods}




\section{Stock populations and experimental conditions}

The experimental populations were established from a long-term laboratory population called Ives (IV) that was initiated from about 200 wild D. melanogaster of each sex collected in Massachusetts in 1975 (Charlesworth \& Charlesworth, 1985). This population has been maintained in the lab at high density, with a census size in thousands, for more than 30 years and is adapted to the laboratory environment (Houle \& Rowe, 2003). In the sexual competition experiment, we also used a reference population homozygous for a recessive ebonymutation previously backcrossed into the IV stock. To estimate pathogen virulence during experimental evolution, at each generation we ran a control using a line homozygous for a recessive relish mutation. The relish mutation blocks the Imd pathway that plays an important role in defense against gram negative bacterial pathogens (Hedengren et al. , 1999)); relish mutants are therefore highly susceptible to P. entomophila (Vallet-Gely et al. , 2010).

All flies in the experiment were maintained on fly media composed of (for $1 \mathrm{~L}$ water): 6.2g Agar powder (ACROS N. 400400050), 58.8g Farigel wheat (Westhove N. FMZH1), 58.8g yeast (Springaline BA10), 100ml grape juice; $4.9 \mathrm{ml}$ Propionic acid (Sigma N. P1386), $26.5 \mathrm{ml}$ of Methyl 4-hydroxybenzoate (Nipagin M, VWR N. ALFAA14289.0) solution $(400 \mathrm{~g} / \mathrm{l})$ in $95 \%$ ethanol. Populations were kept at 250C with a 12L:12D cycle.

Sexual success of infected versus uninfected males

To determine whether infection has any effect on male sexual success, we compared the competitive paternity success of infected and sham-treated males (infection protocol described below). Because the infected and uninfected males came from the same population, we would not be able to distinguish paternity in direct competitions. We therefore competed each against males from a reference population homozygous for the ebony marker.

Each replicate consisted of five focal males (either infected $(\mathrm{N}=38)$ or sham-treated $(\mathrm{N}=39)$ ) and five ebony males competing for fiveebony females. These flies were allowed to interact for 48 hours before being discarded. The resulting offspring were scored upon emergence as adults. The recessive ebony mutation enabled us to distinguish offspring sired by the focal males (which would have wild type cuticles) and those sired by the reference males (which would have dark cuticles). The proportion of wild type offspring was then used as a measure of sexual success of the infected versus non-infected focal males. Even though the fraction of wild type offspring may deviate from the actual fertilization success of focal males because of differences in egg-to-adult survival of wild type and ebony offspring, this would affect the estimates for the two types of males in the same way.

Experimental regimes and selection protocol

To study the interplay between sexual selection (SS) and pathogen presence (P), we used a factorial design that manipulated the presence or absence of SS (polygamous versus monogamous mating systems) and the presence or absence of our pathogen, $P$. entomophila, resulting in 4 experimental regimes $(+\mathrm{SS}+\mathrm{P},+\mathrm{SS}$ $-\mathrm{P}$, -SS $+\mathrm{P},-\mathrm{SS}-\mathrm{P})$. Within each experimental regime, 3 replicate populations were established. Generation 0 adults were obtained by amplifying flies from the IV base population stock, collecting virgin flies, and randomly assigning 80 males and 80 females to each of the 12 populations. At 5-6 days old, virgin males were orally infected with $P$. entomophila (protocol described in the following section) in $+\mathrm{P}$ treatments and sham-infected in $-\mathrm{P}$ treatments. Males were mated with virgin females for 72 hours after being exposed to infection for 24 hours (see below). Under the +SS experimental regimes, groups of 5 males and 5 agematched virgin females were placed in interaction vials. Under the -SS regimes, groups of 1 male and 1 age-matched virgin female were placed in interaction vials. Flies were left in these interaction vials for 72 hours, after which mated females from each population were pooled and re-distributed in groups of 20 to new vials for egg laying. Females were allowed to lay eggs for 72 hours, after which they were discarded from the vials while larvae developed. On emergence, virgins were collected and housed in groups of 20 in single-sex vials until they were 5-6 days old, at which point the experimental protocol was repeated. Populations were maintained under the experimental regimes for 14 generations at a population size of 160 individuals (80 males +80 females). 


\section{Infections}

The pathogen used in our experiments, P. entomophila, is a naturally-occurring gram-negative bacteria isolated from D. melanogaster in Guadeloupe (Vodovar et al. , 2005; Liehl et al. , 2006). It is acquired during feeding and at high doses kills about $60 \%$ of $D$. melanogaster adults within 72 hours and almost $70 \%$ of larvae in 48 hours (Liehl et al. , 2006). It has been found to elicit both local and systemic immune responses involving a range of host responses including the secretion of specific anti-microbial peptides, repair and regeneration of epithelial cells in the gut as a result of damage caused by the pathogen (Vodovar et al. , 2005; Liehl et al. , 2006), and leads to large scale changes in gene expression in response to this pathogen (Chakrabarti et al. , 2012). This system has been used to study the genetic basis of immunity (Chakrabarti et al. , 2012; Neyen et al. , 2014; Bou Sleimanet al. , 2015) as well as in an evolutionary context in work looking at life history trade-offs (Vijendravarma et al. , 2015) and sexual selection (Joye \& Kawecki, 2019).

We obtained an isolate of $P$. entomophila from Bruno Lemaitre (EPFL). Bacteria were plated from glycerol stocks 3 days prior to infection on standard LB-agar plates supplemented with $1 \%$ milk and grown for two days at room temperature. On the day before the infection, a single colony was transferred to a $50 \mathrm{ml}$ Erlenmeyer pre-culture flask with $12.5 \mathrm{ml} \mathrm{LB}$ and incubated for 8 hours in a shaking incubator at $29^{\circ} \mathrm{C}$ and 180rpm. The pre-culture flask was then transferred to a $2 \mathrm{~L}$ Erlenmeyer flask with $400 \mathrm{ml} \mathrm{LB}$ (or 1L Erlenmeyer with $200 \mathrm{ml} \mathrm{LB}$ ) and the culture was incubated overnight in the same shaking incubator at $29^{\circ} \mathrm{C}$ and 180rpm. On the next day, the bacterial culture was centrifuged at $2500 \mathrm{~g}$ at $4^{\circ} \mathrm{C}$ for $20 \mathrm{~min}$. The pellet was re-suspended and mixed with sucrose and water to obtain a final infection cocktail with an OD of 300 . The sham treatment was performed with a $2.5 \%$ sucrose solution.

Oral infection was performed as previously described (Neyen et al. , 2014). Flies were first starved for 4 hours and then transferred to a vial with a filter paper layered over food and soaked with $150 \mu$ of the bacterial cocktail. Males were left in these vials for 24-26 hours after which they were transferred to interaction vials with females. Dead flies were counted at 2, 4, 20 and 24 hours after pathogen exposure.

\section{Bacterial load in infected males}

To examine how fast $D$. melanogaster males clear the $P$. entomophila infection, we infected 1-2 day old virgin males in groups of 20 individuals as described above. We then measured bacterial load of individual flies at 4,8 and $24 \mathrm{~h}$ from the onset of the infection treatment, randomly choosing 2 infection vials to sample at each timepoint. We carefully removed survivors by light anaesthesia and randomly selected 5 individuals.

Each individual fly was then placed in an Eppendorf tube containing small glass beads and $100 \mu \mathrm{L}$ of $70 \%$ ethanol to surface sterilize the fly cuticle. The tube was inverted a few times to ensure proper mixing after which the $70 \%$ ethanol was removed and replaced by $100 \mu \mathrm{L}$ of Luria broth (LB). We then placed the Eppendorf tubes on a Precellys bead ruptor for 30 seconds at 6000rpm in order to homogenize the flies. The homogenate was then serially diluted to obtain concentrations of 1:10, 1:100, 1:1000, 1:10,000 and 1:100,000. We plated $3 \mu \mathrm{L}$ of each of these dilutions in 5 replicates on a single LB plate containing $1 \%$ milk. The plates were left for 50 hours at room temperature and colonies from each dilution and replicate were counted. For each dilution and time point combination, we calculated an average count of the number of colonies for the 5 technical replicates (from each sample) followed by calculating the total colony forming units using the formula below:

Total Colony Forming Units $=$ Number of colonies for a given dilution $\times$ Dilution factor

\section{Survival assays at generation 14}

To assess adaptation to pathogen, two blocks of survival assays were done on males and females after 14 generations of experimental evolution. To avoid parental effects, we first reared individuals from all populations for one generation in a common garden. To establish the common garden, collected virgins were housed together in vials containing 20 males and 20 females. These individuals were allowed to mate for 72 
hours, after which males were discarded. Females ( $\mathrm{N}=120$ per block) from these mating vials were then collected and housed together for 72 hours in groups of 20 to lay eggs. After discarding the females, larvae were allowed to develop and emerging virgin males and females were collected and housed in single-sex groups of 20 each. Virgins (age at infection: Batch 1-5-7 days, Batch 2-4-5 days) were exposed to P. entomophila in the same manner as described above in single-sex groups of 20. After exposure to the pathogen for 24 hours $\left(\mathrm{OD}_{600 \mathrm{~nm}}\right.$ of infections: Batch $1-280$, Batch $\left.2-300\right)$ individuals from each vial were transferred to fresh vials and per vial deaths were scored at 2, 4, 20, 24, 28 (the first time point after transfer to new vials), 44, 52 and 72 hours after pathogen exposure. Alongside the infections, two vials were sham-treated for each of the populations to serve as controls. In each block, we again used flies with a relish mutation to ensure that the pathogen was virulent (Vallet-Gely et al. , 2010).

\section{Statistical analysis}

We performed all statistical analyses in R v3.4.3 with the package afex (Singmann et al. , 2015), a wrapper for lme4 (Bates et al. , 2011). We fit generalised linear mixed models (glmer) with the binomial family (logit link) on counts of either wild type or ebony flies (mating success experiment) or alive and dead flies (survival 72 hours post-infection after 14 generations of experimental evolution) from each vial. For the latter, we fit one model that included all the data (both male and female survival) and included effects of sexual selection, pathogen presence, sex, and all interactions. We also fit simpler models on sex-specific subsets of the data that excluded an effect of sex. In all models, we included experimental block, population, and vial (nested within population) as random effects.

\section{Results}

To assess the potential for sexual selection to act on pathogen resistance, we first compared the paternity success of infected and sham-treated males in competition with males from a reference strain. We found that infected males had lower competitive mating success than uninfected males, as evidenced by a lower proportion of offspring sired by the focal males (treatment effect: $\chi^{2} d f=1=4.45 ; \mathrm{p}=0.03 ;$ Figure 1 , Table 1 ). Infected males sired on average $59.2 \%$ of progeny in competition with the competitive standard, while uninfected males sired on average $68.5 \%$ of progeny. This result indicated that infection harms male mating success and suggested that genetic variation contributing to infection resistance might be favored by sexual selection.

We also verified that the infected males had cleared the pathogen from their gut by the time they were placed with females. Although males harbored many live P. entomophila 4 h after the onset of the infection treatment, no live bacteria were detected at 8 or $24 \mathrm{~h}$ (Supplemental Figure S1), in agreement with earlier results (Bou Sleimanet al. , 2015). Thus, there was little opportunity for the males to transmit the infection to the females.

We next evolved replicate populations with and without both sexual selection and pathogen for fourteen generations. Over the course of experimental evolution, P. entomophila virulence varied; the pathogen reliably killed a substantial fraction of the relishmutants (mean survival post infection $43 \% \pm 10.7$ (s.e.) in relish mutants, Supplemental Figure S2 ). Survival at 24 hours was lower in experimental populations exposed to the pathogen $(+\mathrm{P})$, averaging $92.4 \%$, than it was in populations not exposed to the pathogen $(-\mathrm{P})$, in which survival was $99.9 \%$.

To compare resistance to $P$. entomophila in the populations subject to the different regimes, we measured their survival following infection after fourteen generations of experimental evolution and one generation of common garden rearing. In general, females survived less well after infection than males (Figure 2 ). Populations evolved under pathogen pressure $(+\mathrm{P}$ evolutionary regimes) showed better post-infection survival than populations evolved without pathogen exposure (-P evolutionary regimes) (pathogen selection effect: $\chi_{d f=1}^{2}=8.89 ; \mathrm{p}=0.002 ;$ Figure 2, Table 1$)$. A significant three-way interaction between sexual selection, pathogen, and sex (SS*Pathogen*Sex, $\chi^{2}{ }_{d f=1}=5.91 ; \mathrm{p}=0.01$ ) indicates a difference between males and females in how the interaction between sexual selection and pathogen presence affects post-infection survival, which we further explored in sex-specific analyses. 
In females, the sex-specific analyses showed that post-infection survival under $+\mathrm{P}$ regimes was better than that in the $-\mathrm{P}$ regimes (Figure 2A , pathogen selection effect: $\chi^{2}{ }_{d f=1}=4.92 ; \mathrm{p}=0.026$ ), but we detected no effect of sexual selection $\left(\chi_{d f=1}^{2}=0.04 ; \mathrm{p}=0.82\right)$ or any interaction between sexual selection and pathogen $\left(\chi_{d f=1}^{2}=0.93 ; \mathrm{p}=0.33\right)$. In males there was neither a significant effect of sexual selection (Figure 2B ,$\left.\chi_{d f=1}^{2}=0.094 ; \mathrm{p}=0.75\right)$ nor pathogen $\left(\chi^{2} d f=1=3.40 ; \mathrm{p}=0.06\right)$. However, there was a significant interaction between sexual selection treatment and pathogen presence $\left(\chi^{2}{ }_{d f=1}=4.71 ; \mathrm{p}=0.029\right)$. For the good genes hypothesis to be true in our case, the $+\mathrm{SS}+\mathrm{P}$ populations should have elevated survivorship compared to $-\mathrm{SS}+\mathrm{P}$ regimes. However, in our study we see the opposite effect, with the $-\mathrm{SS}+\mathrm{P}$ regimes surviving significantly better than $+\mathrm{SS}+\mathrm{P}$ (Figure 2B , Tukey's post hoc comparison $\mathrm{p}=0.02$ ). At the same time, there is no difference between +SS populations evolved with and without pathogen. This difference in the effect of sexual selection that depended on whether pathogen was present or not during the course of experimental evolution is what drives the significant interaction between sexual selection and pathogen.

\section{Discussion}

In our study, we aimed to address the interplay of sexual selection and pathogen presence on the evolution of resistance to a pathogen, $P$. entomophila. We found a signature of pathogen resistance in populations evolved under pathogen pressure for fourteen generations when compared to populations evolved without it. Surprisingly, despite only infecting males over the course of experimental evolution, resistance to pathogen was more prominent in females. We did not find any evidence that sexual selection can promote the evolution of resistance to the pathogen, contrary to the predictions of theory (Hamilton \& Zuk, 1982; Westneat \& Birkhead, 1998). We expected that the presence of sexual selection and pathogen pressure would act synergistically, resulting in a greater response to selection and therefore improved survival post-infection. We instead found an antagonistic interaction between the two in males, which could have possibly impeded the evolution of pathogen resistance.

Evolution of increased resistance of $D$. melanogaster to enteric infection and systemic infection has been seen in studies that have experimentally evolved fly populations with P. entomophila (Martins et al. , 2013; Gupta et al. , 2016). The study by Martins et al. (2013) imposed very strong selection on both sexes, with pathogen-induced mortality up to $77 \%$ in the initial generations. In our experiment, pathogen selection was only applied on males and was associated with much lower mortality (5-25\% depending on the generation). This lower virulence likely resulted from a difference in the bacterial genotype and/or the initial Drosophila gene pool; the IV population is generally robust and harbors high levels of genetic variation. It is likely that the overall strength of selection for resistance was therefore considerably lower in our experiment, but yet still sufficient to generate a detectable response. A stronger response to selection might have been obtained with a more virulent pathogen, or if both males and females had been infected each generation. Infecting females introduces a difficulty, however, in that reductions in female mating rate and fecundity make maintenance of experimental populations more challenging, and any reductions in female choosiness due to infection would be expected to diminish the importance of sexual selection.

The fact that females from populations under pathogen pressure evolved higher resistance despite not experiencing direct selection supports a shared genetic basis for immunity between the sexes. Indeed, in line with this idea (Collet et al. , 2016; Connallon \& Hall, 2016), adaptation to desiccation resistance in experimentally evolved populations of $D$. melanogaster was observed both in males and females even when selection was imposed only on males (Gibson Vegaet al. , 2020). Adaptation in our experiment may also be more evident in female post-infection survival simply because females show generally lower survival upon infection relative to males, which would make any evolved differences in survival easier to detect in females than males. Moreover, it is also plausible that alleles contributing to immunity that were favored in males under pathogen pressure had a larger effect size on resistance in females, making female resistance towards pathogen more detectable in this sex. We can exclude the possibility that selection did in fact act directly on females, for example by sexual or social transmission of the pathogen from males to females, because the pathogen was cleared by males by the time they encountered females. 
In our study, we do not see any evidence that sexual selection aids the evolution of resistance to pathogen. Previous studies have attributed the lack of adaptation to novel environments to the negative effects of sexual conflict (Holland \& Rice, 1999b; Rundle et al. , 2006). However, if sexual conflict negatively affected adaptation in our populations we would have expected to find that populations exposed to the pathogen each generation but not experiencing sexual selection $(-\mathrm{SS}+\mathrm{P})$ would show a stronger signal of adaptation to pathogen than those exposed to pathogen and experiencing sexual selection $(+\mathrm{SS}+\mathrm{P})$. While our results on male survival after infection align with this idea, there is no signal of a cost to sexual selection in female survival after infection, leaving it difficult to attribute any importance to sexual conflict in our experiment.

In conclusion, our study found that populations of D. melanogaster evolved resistance to the insect pathogen P. entomophila, but this was either not facilitated (in females) or hindered (in males) by sexual selection. We expect that the low mortality in our study compared to previous work on this pathogen (Guptaet al. , 2013, 2016; Martins et al. , 2013; Joye \& Kawecki, 2019), in which the majority of infected individuals die, provided a level of biological realism. The pathogen was still virulent enough to induce downstream effects on male sexual success, suggesting that genetic variation conferring resistance to pathogen would provide a large target for sexual selection. In addition, because most males survived infection during the course of experimental evolution, this provided an opportunity for sexual selection to reinforce non-sexual selection by magnifying more subtle differences in pathogen resistance (e.g. differences in male condition or vigor that might emerge after weathering the infection). Despite a scenario that seems favorable for the detection of putative benefits of sexual selection - a relatively mild pathogen that might persist in natural host populations, that still yet influences mating success, in a host that harbors genetic variation for resistance - we found no such benefits.

\section{Author contributions}

S.S. and B.H designed experiments, S.S. performed experimental work. S.S. and B.H. analyzed the data. S.S., B.H. and T.J.K. wrote the manuscript.

\section{Acknowledgements}

We would like to thank Bart Deplancke for giving us the lab space to maintain the experimental lines and carry out infections and Loriane Savary for help with survival assays. This work was supported by Swiss National Science Foundation grants PZ00P3_161430 to B.H. and 310030_184791 to T.J.K.

\section{Data Accessibility}

Data will be made available on dryad digital repository on acceptance.

\section{Competing interests}

We declare that we have no competing interests.

\section{References}

Arbuthnott, D. \& Rundle, H.D. 2012. Sexual selection is ineffectual or inhibits the purging of deleterious mutations in drosophila melanogaster. Evolution (N. Y). 66 : 2127-2137.

Armitage, S.A.O. \& Siva-Jothy, M.T. 2005. Immune function responds to selection for cuticular colour in Tenebrio molitor. Heredity (Edinb). 94 : 650-656. Nature Publishing Group.

Bagchi, B., Corbel, Q., Khan, I., Payne, E., Banerji, D., Liljestrand-Rönn, J., et al. 2021. Sexual conflict drives micro- and macroevolution of sexual dimorphism in immunity. BMC Biol.19 : 114. BioMed Central.

Balenger, S.L. \& Zuk, M. 2014. Testing the Hamilton-Zuk hypothesis: Past, present, and future. In: Integrative and Comparative Biology , pp. 601-613. Oxford University Press.

Bates, D., Maechler, M. \& Bolker, B. 2011. lme4: Linear mixed-effects models using S4 classestle.

Blount, J.D., Metcalfe, N.B., Birkhead, T.R. \& Surai, P.F. 2003. Carotenoid modulation of immune function and sexual attractiveness in zebra finches. Science (80-. ). 300 : 125-127. American Association for the 
Advancement of Science.

Bonduriansky, R. \& Chenoweth, S.F. 2009. Intralocus sexual conflict. Elsevier Current Trends.

Bou Sleiman, M.S., Osman, D., Massouras, A., Hoffmann, A.A., Lemaitre, B. \& Deplancke, B. 2015. Genetic, molecular and physiological basis of variation in Drosophila gut immunocompetence. Nat. Commun.6 : 7829. Nature Publishing Group.

Chakrabarti, S., Liehl, P., Buchon, N. \& Lemaitre, B. 2012. Infection-induced host translational blockage inhibits immune responses and epithelial renewal in the Drosophila gut. Cell Host Microbe12 : 60-70.

Chapman, T. 2006. Evolutionary Conflicts of Interest between Males and Females. Cell Press.

Chapman, T., Arnqvist, G., Bangham, J. \& Rowe, L. 2003. Sexual conflict. Trends Ecol. Evol. 18 : 41-47. Elsevier Current Trends.

Charlesworth, B. \& Charlesworth, D. 1985. Genetic variation in recombination in drosophila. I. responses to selection and preliminary genetic analysis. Heredity (Edinb). 54 : 71-83. Nature Publishing Group.

Chippindale, A.K. 2001. Negative genetic correlation for adult fitness between sexes reveals ontogenetic conflict in Drosophila. Proc. Natl. Acad. Sci. 98 : 1671-1675.

Collet, J.M., Fuentes, S., Hesketh, J., Hill, M.S., Innocenti, P., Morrow, E.H., et al. 2016. Rapid evolution of the intersexual genetic correlation for fitness in Drosophila melanogaster.Evolution (N. Y). 70 : 781-795. Evolution.

Connallon, T. \& Hall, M.D. 2016. Genetic correlations and sex-specific adaptation in changing environments. Evolution (N. Y).70 : 2186-2198. Evolution.

Faria, V.G., Martins, N.E., Paulo, T., Teixeira, L., Sucena, É. \& Magalhães, S. 2015. Evolution of Drosophila resistance against different pathogens and infection routes entails no detectable maintenance costs. Evolution (N. Y). $69: 2799-2809$. Society for the Study of Evolution.

Ferro, K., Peuß, R., Yang, W., Rosenstiel, P., Schulenburg, H. \& Kurtz, J. 2019. Experimental evolution of immunological specificity.Proc. Natl. Acad. Sci. U. S. A. 116 : 20598-20604. National Academy of Sciences.

Fisher, R.A. 1930. The genetical theory of natural selection., 2nd ed. Dover, New York, 1958.

Folstad, I. \& Karter, A.J. 1992. Parasites, bright males, and the immunocompetence handicap. Am. Nat. 139 : 603-622. University of Chicago Press.

Fricke, C. \& Arnqvist, G. 2007. Rapid adaptation to a novel host in a seed beetle (Callosobruchus maculatus): The role of sexual selection. Evolution (N. Y). $61: 440-454$.

Getty, T. 2002. Signaling health versus parasites. Am. Nat.159 : 363-371. Am Nat.

Gibson Vega, A., Kennington, W.J., Tomkins, J.L. \& Dugand, R.J. 2020. Experimental evidence for accelerated adaptation to desiccation through sexual selection on males. J. Evol. Biol. 33 : 1060-1067. John Wiley \& Sons, Ltd.

Grieshop, K., Stångberg, J., Martinossi-Allibert, I., Arnqvist, G. \& Berger, D. 2016. Strong sexual selection in males against a mutation load that reduces offspring production in seed beetles. J. Evol. Biol. 29 : 1201-1210. Blackwell Publishing Ltd.

Gupta, V., Ali, Z.S. \& Prasad, N.G. 2013. Sexual activity increases resistance against Pseudomonas entomophila in male Drosophila melanogaster. BMC Evol. Biol. 13.

Gupta, V., Venkatesan, S., Chatterjee, M., Syed, Z.A., Nivsarkar, V. \& Prasad, N.G. 2016. No apparent cost of evolved immune response in Drosophila melanogaster. Evolution (N. Y). 70 : 934-943. Society for the Study of Evolution. 
Hamilton, W.D. \& Zuk, M. 1982. Heritable true fitness and bright birds: A role for parasites? Science (80-. ). $218: 384-387$.

Hangartner, S., Michalczyk, Ł., Gage, M.J.G. \& Martin, O.Y. 2015. Experimental removal of sexual selection leads to decreased investment in an immune component in female Tribolium castaneum. Infect. Genet. Evol. 33 : 212-218. Elsevier.

Hedengren, M., Åsling, B., Dushay, M.S., Ando, I., Ekengren, S., Wihlborg, M., et al. 1999. Relish, a central factor in the control of humoral but not cellular immunity in Drosophila. Mol. Cell 4 : 827-837.

Holland, B. 2002. Sexual selection fails to promote adaptation to a new environment. Evolution (N. Y). 56 : 721-730.

Holland, B. \& Rice, W.R. 1999a. Experimental removal of sexual selection reverses intersexual antagonistic coevolution and removes a reproductive load. Proc. Natl. Acad. Sci. U. S. A. 96 : 5083-5088. National Academy of Sciences.

Holland, B. \& Rice, W.R. 1999b. Experimental removal of sexual selection reverses intersexual antagonistic coevolution and removes a reproductive load. Proc. Natl. Acad. Sci. U. S. A. 96 : 5083-5088. National Academy of Sciences.

Hollis, B., Fierst, J.L. \& Houle, D. 2009. Sexual selection accelerates the elimination of a deleterious mutant in Drosophila melanogaster.Evolution (N. Y). $63: 324-333$.

Hollis, B. \& Houle, D. 2011. Populations with elevated mutation load do not benefit from the operation of sexual selection. J. Evol. Biol. 24 : 1918-1926. NIH Public Access.

Hollis, B., Houle, D., Yan, Z., Kawecki, T.J. \& Keller, L. 2014. Evolution under monogamy feminizes gene expression in Drosophila melanogaster. Nat. Commun. 5 : 3482. Nature Publishing Group.

Hollis, B., Koppik, M., Wensing, K.U., Ruhmann, H., Genzoni, E., Erkosar, B., et al. 2019. Sexual conflict drives male manipulation of female postmating responses in Drosophila melanogaster.Proc. Natl. Acad. Sci. U. S. A. 116 : 8437-8444. National Academy of Sciences.

Hosken, D.J. 2001. Sex and death: Microevolutionary trade-offs between reproductive and immune investment in dung flies. Cell Press.

Hosken, D.J., Archer, C.R. \& Mank, J.E. 2019. Sexual conflict . Press, Princeton University.

Houle, D. \& Kondrashov, A.S. 2002. Coevolution of costly mate choice and condition-dependent display of good genes. Proc. R. Soc. B Biol. Sci. 269 : 97-104. The Royal Society .

Houle, D. \& Rowe, L. 2003. Natural selection in a bottle. Am. Nat. 161 : 50-67. Am Nat.

Hund, A.K., Hubbard, J.K., Albrecht, T., Vortman, Y., Munclinger, P., Krausová, S., et al. 2020. Divergent sexual signals reflect costs of local parasites*. Evolution (N. Y). 74 : 2404-2418. John Wiley \& Sons, Ltd.

Innocenti, P. \& Morrow, E.H. 2010. The sexually antagonistic genes of drosophila melanogaster. PLoS Biol. 8 : e1000335. Public Library of Science.

Iwasa, Y., Pomiankowski, A. \& Nee, S. 1991. The evolution of costly mate preferences. II. The "handicap" principle." Evolution (N. Y). 45 : 1431-1442. John Wiley \& Sons, Ltd.

Jacomb, F., Marsh, J. \& Holman, L. 2016. Sexual selection expedites the evolution of pesticide resistance. Evolution (N. Y). 70 : 2746-2751. Evolution.

Jarzebowska, M. \& Radwan, J. 2010. Sexual selection counteracts extinction of small populations of the bulb mites. Evolution (N. Y). 64 : 1283-1289. John Wiley \& Sons, Ltd.

Joop, G., Roth, O., Schmid-Hempel, P. \& Kurtz, J. 2014. Experimental evolution of external immune defences in the red flour beetle. J. Evol. Biol. 27 : 1562-1571. Blackwell Publishing Ltd. 
Joye, P. \& Kawecki, T.J. 2019. Sexual selection favours good or bad genes for pathogen resistance depending on males' pathogen exposure.Proc. R. Soc. B Biol. Sci. 286 : 20190226. Royal Society Publishing.

Kawecki, T.J., Lenski, R.E., Ebert, D., Hollis, B., Olivieri, I. \& Whitlock, M.C. 2012. Experimental evolution. Elsevier.

Liehl, P., Blight, M., Vodovar, N., Boccard, F. \& Lemaitre, B. 2006. Prevalence of local immune response against oral infection in a Drosophila/Pseudomonas infection model. PLoS Pathog. 2 : 0551-0561. Public Library of Science.

Long, T.A.F., Agrawal, A.F. \& Rowe, L. 2012. The effect of sexual selection on offspring fitness depends on the nature of genetic variation. Curr. Biol. $22: 204-208$.

Long, T.A.F., Pischedda, A., Stewart, A.D. \& Rice, W.R. 2009. A cost of sexual attractiveness to high-fitness females. PLoS Biol.7 : e1000254. Public Library of Science.

Lorch, P.D., Proulx, S., Rowe, L. \& Day, T. 2003. Condition-dependent sexual selection can accelerate adaptation. Evolutionary Ecology.

Lumley, A.J., Michalczyk, Ł., Kitson, J.J.N., Spurgin, L.G., Morrison, C.A., Godwin, J.L., et al. 2015. Sexual selection protects against extinction. Nature 522 : 470-473. Nature Publishing Group.

Marshall Graves, J.A. 2004. The descent of man. Nature427 : 199.

Martin, C. 1990. Parasites and sexual selection : Current Hamilton and Zuk hypothesis. Behav. Ecol. Sociobiol. 328 : 319-328.

Martins, N.E., Faria, V.G., Teixeira, L., Magalhães, S. \& Sucena, É. 2013. Host Adaptation Is Contingent upon the Infection Route Taken by Pathogens. PLoS Pathog. 9 : e1003601. Public Library of Science.

Neyen, C., Bretscher, A.J., Binggeli, O. \& Lemaitre, B. 2014. Methods to study Drosophila immunity. Methods 68 : 116-128. Elsevier Inc.

Parker, G.A. 1979. Sexual selection and sexual conflict. In:Sexual selection and reproductive competition in insects . Academic Press Inc., London.

Price, P.W. 1980. Evolutionary biology of parasites. Princeton University Press, New Jersey.

Promislow, D.E.L., Smith, E.A. \& Pearse, L. 1998. Adult fitness consequences of sexual selection in Drosophila melanogaster. Proc. Natl. Acad. Sci. U. S. A. 95 : 10687-10692.

Radwan, J. 2004. Effectiveness of sexual selection in removing mutations induced with ionizing radiation. Ecol. Lett. 7 : 1149-1154.

Rice, W.R. 1996. Sexually antagonistic male adaptation triggered by experimental arrest of female evolution. Nature 381 : 232-234. Moller, A. P.

Rice, W.R., Stewart, A.D., Morrow, E.H., Linder, J.E., Orteiza, N. \& Byrne, P.G. 2006. Assessing sexual conflict in the Drosophila melanogaster laboratory model system. Philos. Trans. R. Soc. B Biol. Sci. 361 : 287-299. The Royal Society.

Roberts, M.L., Buchanan, K.L. \& Evans, M.R. 2004. Testing the immunocompetence handicap hypothesis: A review of the evidence. Academic Press.

Rundle, H.D., Chenoweth, S.F. \& Blows, M.W. 2006. the Roles of Natural and Sexual Selection During Adaptation To a Novel Environment.Evolution (N. Y). 60 : 2218-2225. Wiley.

Schmid-Hempel, P. 2005. Evolutionary ecology of insect immune defenses.Annu. Rev. Entomol. 50 : $529-551$.

Singh, A., Agrawal, A.F. \& Rundle, H.D. 2017. Environmental complexity and the purging of deleterious alleles. Evolution (N. Y).71 : 2714-2720. Society for the Study of Evolution. 
Singmann, H., Bolker, B. \& Westfall, B. 2015. afex: Analysis of Factorial Experiments. R package version $0.15-2$.

Vallet-Gely, I., Novikov, A., Augusto, L., Liehl, P., Bolbach, G., Péchy-Tarr, M., et al. 2010. Association of hemolytic activity of pseudomonas entomophila, a versatile soil bacterium, with cyclic lipopeptide production. Appl. Environ. Microbiol. 76 : 910-921. American Society for Microbiology.

Van Doorn, G.S. 2009. Intralocus sexual conflict. Ann. N. Y. Acad. Sci. 1168 : 52-71.

Vijendravarma, R.K., Narasimha, S., Chakrabarti, S., Babin, A., Kolly, S., Lemaitre, B., et al. 2015. Gut physiology mediates a trade-off between adaptation to malnutrition and susceptibility to food-borne pathogens. Ecol. Lett. 18 : 1078-1086. Blackwell Publishing Ltd.

Vodovar, N., Vinals, M., Liehl, P., Basset, A., Degrouard, J., Spellman, P., et al. 2005. Drosophila host defense after oral infection by an entomopathogenic Pseudomonas species. Proc. Natl. Acad. Sci. U. S. A. 102 : $11414-11419$.

Westneat, D.F. \& Birkhead, T.R. 1998. Alternative hypotheses linking the immune system and mate choice for good genes. Proc. R. Soc. B Biol. Sci. 265 : 1065-1073. Royal Society.

Whitlock, M.C. \& Agrawal, A.F. 2009. Purging the genome with sexual selection: Reducing mutation load through selection on males. John Wiley \& Sons, Ltd.

Zahavi, A. 1975. Mate selection-A selection for a handicap. J. Theor. Biol. 53 : 205-214. Academic Press.

Figures 


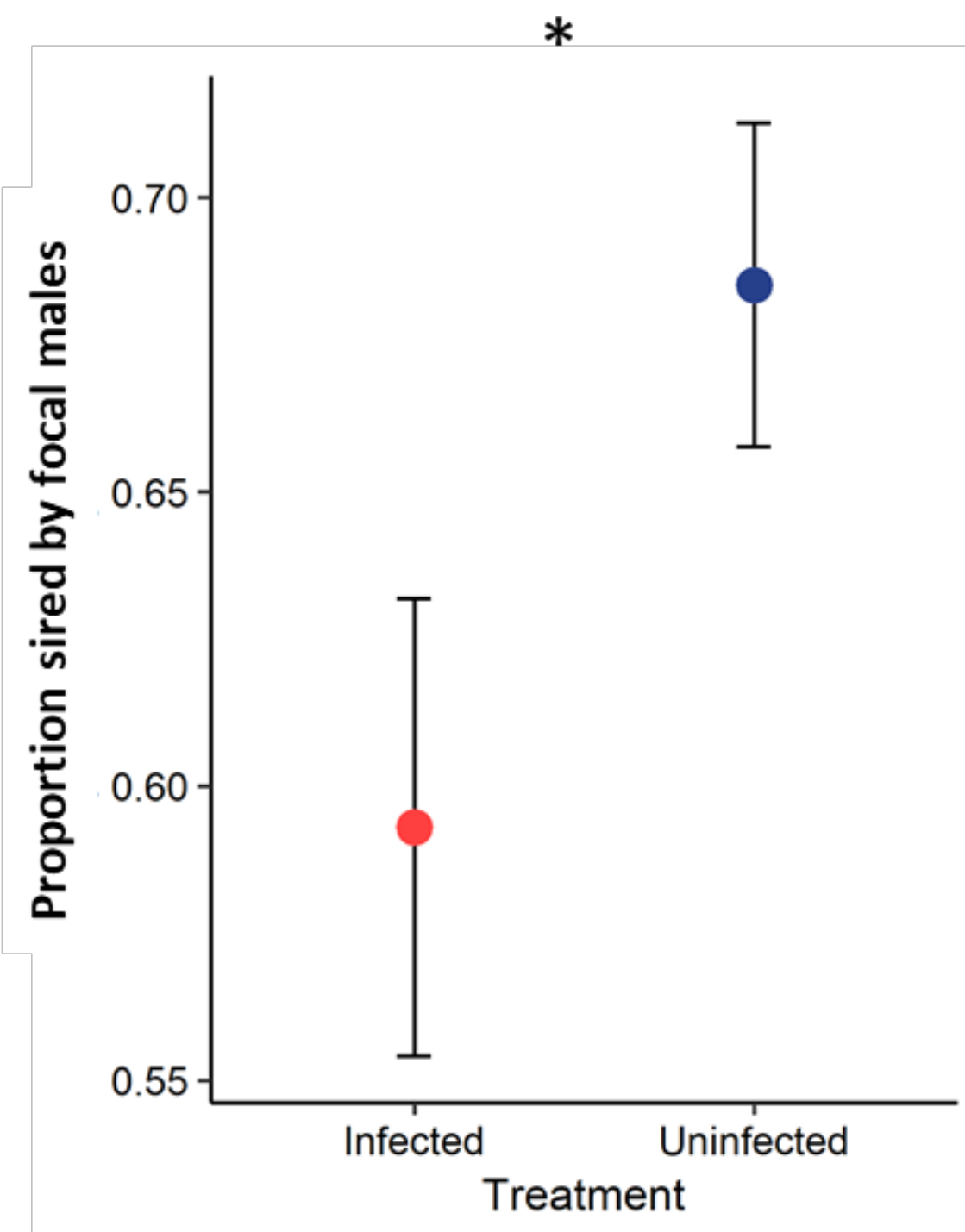

Figure 1 - Competitive reproductive success of infected versus uninfected wild type males in an assay including both pre- and post-copulatory effects. Paternity success is measured relative to a marked standard competitor.

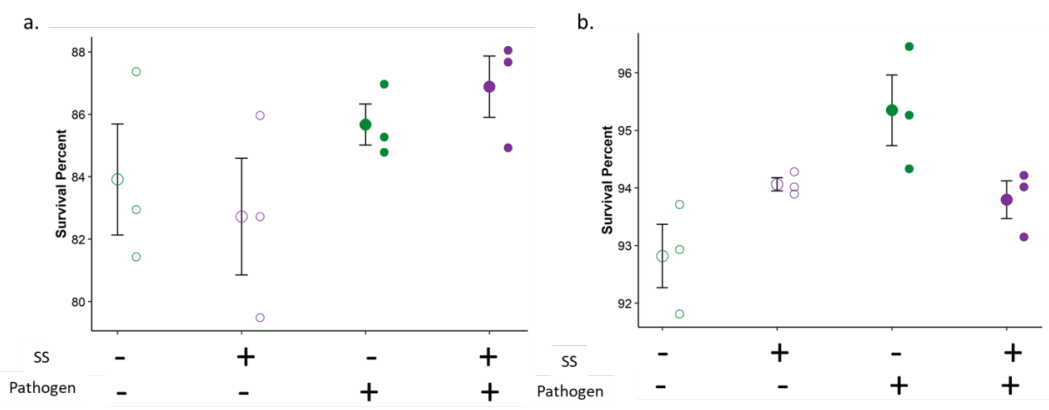


Figure 2 - Survival at 72 hours post infection with P. entomophila, for females (a) and males (b) pooled for both batches. Larger circles indicate the mean $( \pm$ s.e.) of each evolutionary regime, while the smaller points represent the replicate populations within each regime.

Tables

Table 1. Models for survival assays at 72 hours post infection with $P$. entomophila after one generation of common garden rearing after generation 14 .

\begin{tabular}{lllll}
\hline Model & Intercept & $\mathrm{df}$ & $\mathrm{X}^{2}$ & $\mathrm{P}$ \\
\hline Generation 14 & Generation 14 & Generation 14 & Generation 14 & Generation 14 \\
Survival (full model) & & & & \\
SS & -2.24 & 1 & 0.01 & 0.91 \\
Pathogen & & 1 & 8.89 & 0.002 \\
Sex & & 1 & 245.32 & $<2.2 \mathrm{e}^{-16}$ \\
SS:Pathogen & 1 & 1.33 & 0.24 \\
SS:Sex & & 1 & 0.14 & 0.70 \\
Pathogen:Sex & 1 & 0.0074 & 0.93 \\
SS:Pathogen:Sex & & 1 & 5.97 & 0.014 \\
Male Survival & -2.78 & & & \\
Sexual selection & & 1 & 0.094 & 0.75 \\
Pathogen & & 1 & 3.40 & 0.065 \\
Sexual selection x Pathogen & & 1 & 4.71 & 0.029 \\
Female survival & -1.72 & & & \\
Sexual selection & & 1 & 0.04 & 0.82 \\
Pathogen & & 1 & 4.92 & 0.026 \\
Sexual selection x Pathogen & & 1 & 0.93 & 0.33 \\
\hline
\end{tabular}

